\title{
Generalized spaces of double sequences for Orlicz functions and bounded-regular matrices over $n$-normed spaces
}

\author{
Syed Abdul Mohiuddine ${ }^{1 *}$, Kuldip Raj² and Abdullah Alotaibi ${ }^{1}$
}

\section{"Correspondence:}

mohiuddine@gmail.com

'Department of Mathematics,

Faculty of Science, King Abdulaziz

University, P.O. Box 80203, Jeddah,

21589, Saudi Arabia

Full list of author information is

available at the end of the article

\begin{abstract}
The aim of this paper is to introduce some generalized spaces of double sequences with the help of the Musielak-Orlicz function $\mathcal{M}=\left(M_{j k}\right)$ and four-dimensional bounded-regular (shortly, $R H$-regular) matrices $A=\left(a_{n m j k}\right)$ over $n$-normed spaces. Some topological properties and inclusion relations between these spaces are investigated.

MSC: $40 \mathrm{~A} 05 ; 40 \mathrm{D} 25$

Keywords: double sequence; Orlicz function; difference sequence; paranormed space; over $n$-normed spaces; bounded-regular matrices
\end{abstract}

\section{Introduction, notations, and preliminaries}

The concept of 2-normed spaces was first introduced by Gähler [1] in the mid-1960s, while that of $n$-normed spaces one can find in Misiak [2]. Since then, many others have studied this concept and obtained various results; see Gunawan [3, 4] and Gunawan and Mashadi [5]. Let $n \in \mathbb{N}$ and $X$ be a linear space over the field of real numbers $\mathbb{R}$ of dimension $d$, where $d \geq n \geq 2$. A real valued function $\|\cdot, \ldots, \cdot\|$ on $X^{n}$ satisfying the following four conditions:

(1) $\left\|x_{1}, x_{2}, \ldots, x_{n}\right\|=0$ if and only if $x_{1}, x_{2}, \ldots, x_{n}$ are linearly dependent in $X$,

(2) $\left\|x_{1}, x_{2}, \ldots, x_{n}\right\|$ is invariant under permutation,

(3) $\left\|\alpha x_{1}, x_{2}, \ldots, x_{n}\right\|=|\alpha|\left\|x_{1}, x_{2}, \ldots, x_{n}\right\|$ for any $\alpha \in \mathbb{R}$, and

(4) $\left\|x+x^{\prime}, x_{2}, \ldots, x_{n}\right\| \leq\left\|x, x_{2}, \ldots, x_{n}\right\|+\left\|x^{\prime}, x_{2}, \ldots, x_{n}\right\|$

is called an $n$-norm on $X$, and the pair $(X,\|\cdot, \ldots, \cdot\|)$ is called a $n$-normed space over the field $\mathbb{R}$.

For example, we may take $X=\mathbb{R}^{n}$ being equipped with the $n$-norm $\left\|x_{1}, x_{2}, \ldots, x_{n}\right\|_{E}=$ the volume of the $n$-dimensional parallelepiped spanned by the vectors $x_{1}, x_{2}, \ldots, x_{n}$ which may be given explicitly by the formula

$$
\left\|x_{1}, x_{2}, \ldots, x_{n}\right\|_{E}=\left|\operatorname{det}\left(x_{i j}\right)\right|,
$$

where $x_{i}=\left(x_{i 1}, x_{i 2}, \ldots, x_{i n}\right) \in \mathbb{R}^{n}$ for each $i=1,2, \ldots, n$. Let $(X,\|\cdot, \ldots, \cdot\|)$ be an $n$-normed space of dimension $d \geq n \geq 2$ and $\left\{a_{1}, a_{2}, \ldots, a_{n}\right\}$ be a linearly independent set in $X$. Then

(c) 2014 Mohiuddine et al.; licensee Springer. This is an Open Access article distributed under the terms of the Creative Commons Attribution License (http://creativecommons.org/licenses/by/2.0), which permits unrestricted use, distribution, and reproduction in any medium, provided the original work is properly cited. 
the function $\|\cdot, \ldots, \cdot\|_{\infty}$ on $X^{n-1}$ defined by

$$
\left\|x_{1}, x_{2}, \ldots, x_{n-1}\right\|_{\infty}=\max \left\{\left\|x_{1}, x_{2}, \ldots, x_{n-1}, a_{i}\right\|: i=1,2, \ldots, n\right\}
$$

defines an $(n-1)$-norm on $X$ with respect to $\left\{a_{1}, a_{2}, \ldots, a_{n}\right\}$.

A sequence $\left(x_{k}\right)$ in a $n$-normed space $(X,\|\cdot, \ldots, \cdot\|)$ is said to converge to some $L \in X$ if

$$
\lim _{k \rightarrow \infty}\left\|x_{k}-L, z_{1}, \ldots, z_{n-1}\right\|=0 \quad \text { for every } z_{1}, \ldots, z_{n-1} \in X
$$

A sequence $\left(x_{k}\right)$ in a $n$-normed space $(X,\|\cdot, \ldots, \cdot\|)$ is said to be Cauchy if

$$
\lim _{k, p \rightarrow \infty}\left\|x_{k}-x_{p}, z_{1}, \ldots, z_{n-1}\right\|=0 \quad \text { for every } z_{1}, \ldots, z_{n-1} \in X
$$

If every Cauchy sequence in $X$ converges to some $L \in X$, then $X$ is said to be complete with respect to the $n$-norm. A complete $n$-normed space is called $n$-Banach space.

Of the definitions of convergence commonly employed for double series, only that due to Pringsheim permits a series to converge conditionally. Therefore, in spite of any disadvantages which it may possess, this definition is better adapted than others to the study of many problems in double sequences and series. Chief among the reasons why the theory of double sequences, under the Pringsheim definition of convergence, presents difficulties not encountered in the theory of simple sequences is the fact that a double sequence $\left\{x_{i j}\right\}$ may converge without $x_{i j}$ being a bounded function of $i$ and $j$. Thus it is not surprising that many authors in dealing with the convergence of double sequences should have restricted themselves to the class of bounded sequences or, in dealing with the summability of double series, to the class of series for which the function whose limit is the sum of the series is a bounded function of $i$ and $j$. Without such a restriction, peculiar things may sometimes happen; for example, a double power series may converge with partial sum $\left\{S_{i j}\right\}$ unbounded at a place exterior to its associated circles of convergence. Nevertheless there are problems in the theory of double sequences and series where this restriction of boundedness as it has been applied is considerably more stringent than need be. In [6], Hardy introduced the concept of regular convergence for double sequences. Some important work on double sequences has also been done by Bromwich [7]. Later on, it was studied by various authors, e.g. Móricz [8], Móricz and Rhoades [9], Başarır and Sonalcan [10], Mursaleen and Mohiuddine [11, 12], and many others. Mursaleen [13] has defined and characterized the notion of almost strong regularity of four-dimensional matrices and applied these matrices to establish a core theorem (also see [14]). Altay and Başar [15] have recently introduced the double sequence spaces $\mathcal{B S}, \mathcal{B S}(t), \mathcal{C} \mathcal{S}_{p}, \mathcal{C S}_{b p}, \mathcal{C} \mathcal{S}_{r}$, and $\mathcal{B V}$ consisting of all double series whose sequence of partial sums are in the spaces $\mathcal{M}_{u}$, $\mathcal{M}_{u}(t), \mathcal{C}_{p}, \mathcal{C}_{b p}, \mathcal{C}_{r}$, and $\mathcal{L}_{u}$, respectively. Başar and Sever [16] extended the well known space $\ell_{q}$ from single sequence to double sequences, denoted by $\mathcal{L}_{q}$, and established its interesting properties. The authors of [17] defined some convex and paranormed sequences spaces and presented some interesting characterization. Most recently, Mohiuddine and Alotaibi [18] introduced some new double sequences spaces for $\sigma$-convergence of double sequences and invariant mean, and also determined some inclusion results for these spaces. For more details on these concepts, one is referred to [19-21]. 
The notion of difference sequence spaces was introduced by Kizmaz [22], who studied the difference sequence spaces $l_{\infty}(\Delta), c(\Delta)$, and $c_{0}(\Delta)$. The notion was further generalized by Et and Çolak [23] by introducing the spaces $l_{\infty}\left(\Delta^{r}\right), c\left(\Delta^{r}\right)$, and $c_{0}\left(\Delta^{r}\right)$.

Let $w$ be the space of all complex or real sequences $x=\left(x_{k}\right)$ and let $r$ and $s$ be two nonnegative integers. Then for $Z=l_{\infty}, c, c_{0}$, we have the following sequence spaces:

$$
Z\left(\Delta_{s}^{r}\right)=\left\{x=\left(x_{k}\right) \in w:\left(\Delta_{s}^{r} x_{k}\right) \in Z\right\},
$$

where $\Delta_{s}^{r} x=\left(\Delta_{s}^{r} x_{k}\right)=\left(\Delta_{s}^{r-1} x_{k}-\Delta_{s}^{r-1} x_{k+1}\right)$ and $\Delta^{0} x_{k}=x_{k}$ for all $k \in \mathbb{N}$, which is equivalent to the following binomial representation:

$$
\Delta_{s}^{r} x_{k}=\sum_{v=0}^{r}(-1)^{v}\left(\begin{array}{l}
r \\
v
\end{array}\right) x_{k+s v} .
$$

We remark that for $s=1$ and $r=s=1$, we obtain the sequence spaces which were introduced and studied by Et and Çolak [23] and Kızmaz [22], respectively. For more details as regards sequence spaces, see [24-31] and references therein.

An Orlicz function $M:[0, \infty) \rightarrow[0, \infty)$ is a continuous, nondecreasing, and convex such that $M(0)=0, M(x)>0$ for $x>0$ and $M(x) \rightarrow \infty$ as $x \rightarrow \infty$. If convexity of the Orlicz function is replaced by $M(x+y) \leq M(x)+M(y)$, then this function is called modulus function. Lindenstrauss and Tzafriri [32] used the idea of Orlicz function to define the sequence space

$$
\ell_{M}=\left\{x=\left(x_{k}\right) \in w: \sum_{k=1}^{\infty} M\left(\frac{\left|x_{k}\right|}{\rho}\right)<\infty, \text { for some } \rho>0\right\},
$$

known as an Orlicz sequence space. The space $\ell_{M}$ is a Banach space with the norm

$$
\|x\|=\inf \left\{\rho>0: \sum_{k=1}^{\infty} M\left(\frac{\left|x_{k}\right|}{\rho}\right) \leq 1\right\} .
$$

Also it was shown in [32] that every Orlicz sequence space $\ell_{M}$ contains a subspace isomorphic to $\ell_{p}(p \geq 1)$. An Orlicz function $M$ can always be represented in the integral form

$$
M(x)=\int_{0}^{x} \eta(t) d t
$$

where $\eta$ is known as the kernel of $M$, is a right differentiable for $t \geq 0, \eta(0)=0, \eta(t)>0, \eta$ is nondecreasing and $\eta(t) \rightarrow \infty$ as $t \rightarrow \infty$.

A sequence $\mathcal{M}=\left(M_{k}\right)$ of Orlicz functions is said to be Musielak-Orlicz function (see $[33,34])$. A sequence $\mathcal{N}=\left(N_{k}\right)$ is defined by

$$
N_{k}(v)=\sup \left\{|v| u-M_{k}(u): u \geq 0\right\}, \quad k=1,2, \ldots
$$

is called the complementary function of a Musielak-Orlicz function $\mathcal{M}$. For a given Musielak-Orlicz function $\mathcal{M}$, the Musielak-Orlicz sequence space $t_{\mathcal{M}}$ and its subspace 
$h_{\mathcal{M}}$ are defined as follows:

$$
\begin{aligned}
& t_{\mathcal{M}}=\left\{x \in w: I_{\mathcal{M}}(c x)<\infty \text { for some } c>0\right\}, \\
& h_{\mathcal{M}}=\left\{x \in w: I_{\mathcal{M}}(c x)<\infty \text { for all } c>0\right\},
\end{aligned}
$$

where $I_{\mathcal{M}}$ is a convex modular defined by

$$
I_{\mathcal{M}}(x)=\sum_{k=1}^{\infty} M_{k}\left(x_{k}\right), \quad x=\left(x_{k}\right) \in t_{\mathcal{M}}
$$

We consider $t_{\mathcal{M}}$ equipped with the Luxemburg norm

$$
\|x\|=\inf \left\{k>0: I_{\mathcal{M}}\left(\frac{x}{k}\right) \leq 1\right\}
$$

or equipped with the Orlicz norm

$$
\|x\|^{0}=\inf \left\{\frac{1}{k}\left(1+I_{\mathcal{M}}(k x)\right): k>0\right\}
$$

A Musielak-Orlicz function $\mathcal{M}=\left(M_{k}\right)$ is said to satisfy the $\Delta_{2}$-condition if there exist constants $a, K>0$, and a sequence $c=\left(c_{k}\right)_{k=1}^{\infty} \in l_{+}^{1}$ (the positive cone of $l^{1}$ ) such that the inequality

$$
M_{k}(2 u) \leq K M_{k}(u)+c_{k}
$$

holds for all $k \in \mathbb{N}$ and $u \in \mathbb{R}^{+}$, whenever $M_{k}(u) \leq a$.

A double sequence $x=\left(x_{j k}\right)$ is said to be bounded if $\|x\|_{(\infty, 2)}=\sup _{j, k}\left|x_{j k}\right|<\infty$. We denote by $l_{\infty}^{2}$, the space of all bounded double sequences.

By the convergence of double sequence $x=\left(x_{j k}\right)$ we mean the convergence in the Pringsheim sense i.e. a double sequence $x=\left(x_{j k}\right)$ is said to converge to the limit $L$ in Pringsheim sense (denoted by $P-\lim x=L$ ) provided that given $\epsilon>0$ there exists $n \in \mathbb{N}$ such that $\left|x_{j k}-L\right|<\epsilon$ whenever $j, k>n$ (see [35]). We shall write more briefly as $P$-convergent. If, in addition, $x \in l_{\infty}^{2}$, then $x$ is said to be boundedly P-convergent to $L$. We shall denote the space of all bounded convergent double sequences (or, boundedly $P$-convergent) by $c_{\infty}^{2}$.

Let $S \subseteq \mathbb{N} \times \mathbb{N}$ and let $\epsilon>0$ be given. By $\chi_{S(x ; \epsilon)}$, we denote the characteristic function of the set $S(x ; \epsilon)=\left\{(j, k) \in \mathbb{N} \times \mathbb{N}:\left|x_{j k}\right| \geq \epsilon\right\}$.

Let $A=\left(a_{n m j k}\right)$ be a four-dimensional infinite matrix of scalars. For all $m, n \in \mathbb{N}_{0}$, where $\mathbb{N}_{0}:=\mathbb{N} \cup\{0\}$, the sum

$$
y_{n m}=\sum_{j, k=0,0}^{\infty, \infty} a_{n m j k} x_{j k}
$$

is called the $A$-means of the double sequence $\left(x_{j k}\right)$. A double sequence $\left(x_{j k}\right)$ is said to be $A$-summable to the limit $L$ if the $A$-means exist for all $m, n$ in the sense of Pringsheim's convergence:

$$
P-\lim _{p, q \rightarrow \infty} \sum_{j, k=0,0}^{p, q} a_{n m j k} x_{j k}=y_{n m} \quad \text { and } \quad P-\lim _{n, m \rightarrow \infty} y_{n m}=L .
$$


A four-dimensional matrix $A$ is said to be bounded-regular (or $R H$-regular) if every bounded $P$-convergent sequence is $A$-summable to the same limit and the $A$-means are also bounded.

The following is a four-dimensional analog of the well-known Silverman-Toeplitz theorem [36].

Theorem 1.1 (Robison [37] and Hamilton [38]) The four-dimensional matrix $A$ is $R H$ regular if and only if

$\left(\mathrm{RH}_{1}\right) P$ - $\lim _{n, m} a_{n m j k}=0$ for each $j$ and $k$,

$\left(\mathrm{RH}_{2}\right) P-\lim _{n, m} \sum_{j, k=0,0}^{\infty, \infty}\left|a_{n m j k}\right|=1$,

$\left(\mathrm{RH}_{3}\right) P-\lim _{n, m} \sum_{j=0}^{\infty}\left|a_{n m j k}\right|=0$ for each $k$,

$\left(\mathrm{RH}_{4}\right) P-\lim _{n, m} \sum_{k=0}^{\infty}\left|a_{n m j k}\right|=0$ for each $j$,

$\left(\mathrm{RH}_{5}\right) \sum_{j, k=0,0}^{\infty, \infty}\left|a_{n m j k}\right|<\infty$ for all $n, m \in \mathbb{N}_{0}$.

\section{Some spaces of double sequences over $\boldsymbol{n}$-normed spaces}

Recently, Yurdakadim and Tas [41] defined the spaces of double sequences for RH-regular four-dimensional matrices and Orlicz functions and also established some interesting results. Quite recently, Mohiuddine et al. [42] defined and studied some paranormed double difference sequence spaces for four-dimensional bounded-regular matrices and MusielakOrlicz functions.

Recall that a linear topological space $X$ over the real field $\mathbb{R}$ (the set of real numbers) is said to be a paranormed space if there is a subadditive function $g: X \rightarrow \mathbb{R}$ such that $g(\theta)=0, g(x)=g(-x)$ and scalar multiplication is continuous, i.e., $\left|\alpha_{n}-\alpha\right| \rightarrow 0$ and $g\left(x_{n}-\right.$ $x) \rightarrow 0$ imply $g\left(\alpha_{n} x_{n}-\alpha x\right) \rightarrow 0$ for all $\alpha$ 's in $\mathbb{R}$ and all $x$ 's in $X$, where $\theta$ is the zero vector in the linear space $X$.

The linear spaces $l_{\infty}(p), c(p), c_{0}(p)$ were defined by Maddox [39] (also, see Simons [40]). Let $(X,\|\cdot, \ldots, \cdot\|)$ be a $n$-normed space and $w(n-X)$ denotes the space of $X$-valued sequences. Let $\mathcal{M}=\left(M_{j k}\right)$ be a Musielak-Orlicz function, that is, $\mathcal{M}$ is a sequence of Orlicz functions and let $A=\left(a_{n m j k}\right)$ be a nonnegative four-dimensional bounded-regular matrix. Then we define the following double difference sequence spaces over $n$-normed spaces:

$$
\begin{aligned}
& W_{0}^{2}\left(A, \mathcal{M}, u, \Delta_{s}^{r}, p,\|\cdot, \ldots, \cdot\|\right) \\
& =\left\{x=\left(x_{j k}\right) \in w(n-X): P-\lim _{n, m} \frac{1}{n m} \sum_{j, k=0,0}^{\infty, \infty} a_{n m j k}\left[M_{j k}\left(\left\|\frac{u_{j k} \Delta_{s}^{r} x_{j k}}{\rho}, z_{1}, \ldots, z_{n-1}\right\|\right)^{p_{j k}}\right]\right. \\
& \quad=0 \text { for some } \rho>0\}
\end{aligned}
$$

and

$$
\begin{gathered}
W^{2}\left(A, \mathcal{M}, u, \Delta_{s}^{r}, p,\|\cdot, \ldots, \cdot\|\right) \\
=\left\{x=\left(x_{j k}\right) \in w(n-X):\right.
\end{gathered}
$$




$$
\begin{aligned}
& P-\lim _{n, m} \frac{1}{n m} \sum_{j, k=0,0}^{\infty, \infty} a_{n m j k}\left[M_{j k}\left(\left\|\frac{u_{j k} \Delta_{s}^{r} x_{j k}-L}{\rho}, z_{1}, \ldots, z_{n-1}\right\|\right)^{p_{j k}}\right] \\
& =0 \text { for some } \rho>0 \text { and } L \in \mathbb{C}\},
\end{aligned}
$$

where $p=\left(p_{j k}\right)$ is a double sequence of real numbers such that $p_{j k}>0$ for $j, k$ and $\sup _{j, k} p_{j k}=H<\infty$, and $u=\left(u_{j k}\right)$ is a double sequence of strictly positive real numbers.

We obtain the following sequence spaces from the above sequence spaces: $W_{0}^{2}(A, \mathcal{M}, u$, $\left.\Delta_{s}^{r}, p,\|\cdot, \ldots, \cdot\|\right)$ and $W^{2}\left(A, \mathcal{M}, u, \Delta_{s}^{r}, p,\|\cdot, \ldots, \cdot\|\right)$ by giving particular values to $\mathcal{M}, p, u$, and $A$.

(i) If $\mathcal{M}(x)=x$, then we write $W_{0}^{2}\left(A, u, \Delta_{s}^{r}, p,\|\cdot, \ldots, \cdot\|\right)$ and $W^{2}\left(A, u, \Delta_{s}^{r}, p,\|\cdot, \ldots, \cdot\|\right)$ instead of $W_{0}^{2}\left(A, \mathcal{M}, u, \Delta_{s}^{r}, p,\|\cdot, \ldots, \cdot\|\right)$ and $W^{2}\left(A, \mathcal{M}, u, \Delta_{s}^{r}, p,\|\cdot, \ldots, \cdot\|\right)$, respectively.

(ii) If $p=\left(p_{j k}\right)=1$ for all $j, k$, then we write $W_{0}^{2}\left(A, \mathcal{M}, u, \Delta_{s}^{r},\|\cdot, \ldots, \cdot\|\right)$ and $W^{2}(A, \mathcal{M}, u$, $\left.\Delta_{s}^{r},\|\cdot, \ldots, \cdot\|\right)$ instead of $W_{0}^{2}\left(A, \mathcal{M}, u, \Delta_{s}^{r}, p,\|\cdot, \ldots, \cdot\|\right)$ and $W^{2}\left(A, \mathcal{M}, u, \Delta_{s}^{r}, p\right.$, $\|\cdot, \ldots, \cdot\|)$, respectively.

(iii) If $u=\left(u_{j k}\right)=1$ for all $j, k$, then we write $W_{0}^{2}\left(A, \mathcal{M}, \Delta_{s}^{r}, p,\|\cdot, \ldots, \cdot\|\right)$ and $W^{2}(A, \mathcal{M}$, $\left.\Delta_{s}^{r}, p,\|\cdot, \ldots, \cdot\|\right)$ instead of $W_{0}^{2}\left(A, \mathcal{M}, u, \Delta_{s}^{r}, p,\|\cdot, \ldots, \cdot\|\right)$ and $W^{2}\left(A, \mathcal{M}, u, \Delta_{s}^{r}, p\right.$, $\|\cdot, \ldots, \cdot\|)$, respectively.

(iv) If $A=(C, 1,1)$, then we write $W_{0}^{2}\left(\mathcal{M}, u, \Delta_{s}^{r}, p,\|\cdot, \ldots, \cdot\|\right)$ and $W^{2}\left(\mathcal{M}, u, \Delta_{s}^{r}, p\right.$, $\|\cdot, \ldots, \cdot\|)$ instead of $W_{0}^{2}\left(A, \mathcal{M}, u, \Delta_{s}^{r}, p,\|\cdot, \ldots, \cdot\|\right)$ and $W^{2}\left(A, \mathcal{M}, u, \Delta_{s}^{r}, p,\|\cdot, \ldots, \cdot\|\right)$, respectively, where $(C, 1,1)$ denotes the $n m$ th Cesàro mean of double sequence $\left(x_{j k}\right)$.

(v) If $A=(C, 1,1)$ and $\mathcal{M}(x)=x$, then we write $W_{0}^{2}\left(u, \Delta_{s}^{r}, p,\|\cdot, \ldots, \cdot\|\right)$ and $W^{2}\left(u, \Delta_{s}^{r}, p\right.$, $\|\cdot, \ldots, \cdot\|)$ instead of $W_{0}^{2}\left(A, \mathcal{M}, u, \Delta_{s}^{r}, p,\|\cdot, \ldots, \cdot\|\right)$ and $W^{2}\left(A, \mathcal{M}, u, \Delta_{s}^{r}, p,\|\cdot, \ldots, \cdot\|\right)$, respectively.

Throughout the paper, we shall use the following inequality: Let $\left(a_{j k}\right)$ and $\left(b_{j k}\right)$ be two double sequences. Then

$$
\left|a_{j k}+b_{j k}\right|^{p_{j k}} \leq K\left(\left|a_{j k}\right|^{p_{j k}}+\left|b_{j k}\right|^{p_{j k}}\right)
$$

where $K=\max \left(1,2^{H-1}\right)$ and $\sup _{j, k} p_{j k}=H($ see $[18])$.

\section{Main results}

Theorem 3.1 Let $\mathcal{M}=\left(M_{j k}\right)$ be a Musielak-Orlicz function, $A=\left(a_{n m j k}\right)$ be a nonnegative four-dimensional RH-regular matrix, $p=\left(p_{j k}\right)$ be a bounded sequence of positive real numbers and $u=\left(u_{j k}\right)$ be a sequence of strictly positive real numbers. Then $W_{0}^{2}\left(A, \mathcal{M}, u, \Delta_{s}^{r}, p,\|\cdot, \ldots, \cdot\|\right)$ and $W^{2}\left(A, \mathcal{M}, u, \Delta_{s}^{r}, p,\|\cdot, \ldots, \cdot\|\right)$ are linear spaces over the field $\mathbb{R}$ of reals.

Proof Suppose $x=\left(x_{j k}\right)$ and $y=\left(y_{j k}\right) \in W_{0}^{2}\left(A, \mathcal{M}, u, \Delta_{s}^{r}, p,\|\cdot, \ldots, \cdot\|\right)$ and $\alpha, \beta \in \mathbb{R}$. Then there exist positive numbers $\rho_{1}, \rho_{2}$ such that

$$
\lim _{n, m} \frac{1}{n m} \sum_{j, k=0,0}^{\infty, \infty} a_{n m j k}\left[M_{j k}\left(\left\|\frac{u_{j k} \Delta_{s}^{r} x_{j k}}{\rho_{1}}, z_{1}, \ldots, z_{n-1}\right\|\right)^{p_{j k}}\right]=0 \quad \text { for some } \rho_{1}>0
$$


and

$$
\lim _{n, m} \frac{1}{n m} \sum_{j, k=0,0}^{\infty, \infty} a_{n m j k}\left[M_{j k}\left(\left\|\frac{u_{j k} \Delta_{s}^{r} y_{j k}}{\rho_{2}}, z_{1}, \ldots, z_{n-1}\right\|\right)^{p_{j k}}\right]=0 \quad \text { for some } \rho_{2}>0
$$

Let $\rho_{3}=\max \left(2|\alpha| \rho_{1}, 2|\beta| \rho_{2}\right)$. Since $\mathcal{M}=\left(M_{j k}\right)$ is a nondecreasing and convex so by using inequality (2.1), we have

$$
\begin{aligned}
\lim _{n, m} \frac{1}{n m} \sum_{j, k=0,0}^{\infty, \infty} a_{n m j k}\left[M_{j k}\left(\left\|\frac{u_{j k} \Delta_{s}^{r}\left(\alpha x_{j k}+\beta y_{j k}\right)}{\rho_{3}}, z_{1}, \ldots, z_{n-1}\right\|\right)^{p_{j k}}\right] \\
\leq K \lim _{n, m} \frac{1}{n m} \sum_{j, k=0,0}^{\infty, \infty} \frac{1}{2^{p_{j k}}} a_{n m j k}\left[M_{j k}\left(\left\|\frac{u_{j k} \Delta_{s}^{r} x_{j k}}{\rho_{1}}, z_{1}, \ldots, z_{n-1}\right\|\right)^{p_{j k}}\right] \\
\quad+K \lim _{n, m} \frac{1}{n m} \sum_{j, k=0,0}^{\infty, \infty} \frac{1}{2^{p_{j k}}} a_{n m j k}\left[M_{j k}\left(\left\|\frac{u_{j k} \Delta_{s}^{r} y_{j k}}{\rho_{2}}, z_{1}, \ldots, z_{n-1}\right\|\right)^{p_{j k}}\right] \\
\leq K \lim _{n, m} \frac{1}{n m} \sum_{j, k=0,0}^{\infty, \infty} a_{n m j k}\left[M_{j k}\left(\left\|\frac{u_{j k} \Delta_{s}^{r} x_{j k}}{\rho_{1}}, z_{1}, \ldots, z_{n-1}\right\|\right)^{p_{j k}}\right] \\
\quad+K \lim _{n, m} \frac{1}{n m} \sum_{j, k=0,0}^{\infty, \infty} a_{n m j k}\left[M_{j k}\left(\left\|\frac{u_{j k} \Delta_{s}^{r} y_{j k}}{\rho_{2}}, z_{1}, \ldots, z_{n-1}\right\|\right)^{p_{j k}}\right] \\
=0 .
\end{aligned}
$$

Thus $\alpha x+\beta y \in W_{0}^{2}\left(A, \mathcal{M}, u, \Delta_{s}^{r}, p,\|\cdot, \ldots, \cdot\|\right)$. This proves that $W_{0}^{2}\left(A, \mathcal{M}, u, \Delta_{s}^{r}, p,\|\cdot, \ldots, \cdot\|\right)$ is a linear space. Similarly we can prove that $W^{2}\left(A, \mathcal{M}, u, \Delta_{s}^{r}, p,\|\cdot, \ldots, \cdot\|\right)$ is also a linear space.

Theorem 3.2 Let $\mathcal{M}=\left(M_{j k}\right)$ be a Musielak-Orlicz function, $A=\left(a_{n m j k}\right)$ be a nonnegative four-dimensional RH-regular matrix, $p=\left(p_{j k}\right)$ be a bounded sequence of positive real numbers and $u=\left(u_{j k}\right)$ be a sequence of strictly positive real numbers. Then $W_{0}^{2}\left(A, \mathcal{M}, u, \Delta_{s}^{r}, p,\|\cdot, \ldots, \cdot\|\right)$ and $W^{2}\left(A, \mathcal{M}, u, \Delta_{s}^{r}, p,\|\cdot, \ldots, \cdot\|\right)$ are paranormed spaces with the paranorm

$$
\begin{aligned}
g(x)= & \inf \left\{(\rho)^{\frac{p_{j k}}{M}}: \lim _{n, m} \frac{1}{n m} \sum_{j, k=0,0}^{\infty, \infty} a_{n m j k}\left[M_{j k}\left(\left\|\frac{u_{j k} \Delta_{s}^{r} x_{j k}}{\rho}, z_{1}, \ldots, z_{n-1}\right\|\right)^{p_{j k}}\right]^{\frac{1}{M}}\right. \\
& \leq 1 \text { for some } \rho>0\},
\end{aligned}
$$

where $0<p_{j k} \leq \sup p_{j k}=H<\infty$ and $M=\max (1, H)$.

Proof (i) Clearly $g(x) \geq 0$ for $x=\left(x_{j k}\right) \in W_{0}^{2}\left(A, \mathcal{M}, u, \Delta_{s}^{r}, p,\|\cdot, \ldots, \cdot\|\right)$. Since $M_{j k}(0)=0$, we get $g(0)=0$.

(ii) $g(-x)=g(x)$. 
Mohiuddine et al. Journal of Inequalities and Applications 2014, 2014:332

Page 8 of 16

http://www.journalofinequalitiesandapplications.com/content/2014/1/332

(iii) Let $x=\left(x_{j k}\right), y=\left(y_{j k}\right) \in W_{0}^{2}\left(A, \mathcal{M}, u, \Delta_{s}^{r}, p,\|\cdot, \ldots, \cdot\|\right)$ there exist positive numbers $\rho_{1}$ and $\rho_{2}$ such that

$$
\lim _{n, m} \frac{1}{n m} \sum_{j, k=0,0}^{\infty, \infty} a_{n m j k}\left[M_{j k}\left(\left\|\frac{u_{j k} \Delta_{s}^{r} x_{j k}}{\rho_{1}}, z_{1}, \ldots, z_{n-1}\right\|\right)^{p_{j k}}\right] \leq 1
$$

and

$$
\lim _{n, m} \frac{1}{n m} \sum_{j, k=0,0}^{\infty, \infty} a_{n m j k}\left[M_{j k}\left(\left\|\frac{u_{j k} \Delta_{s}^{r} y_{j k}}{\rho_{2}}, z_{1}, \ldots, z_{n-1}\right\|\right)^{p_{j k}}\right] \leq 1
$$

Let $\rho=\rho_{1}+\rho_{2}$. Then by using Minkowski's inequality, we have

$$
\begin{aligned}
& \lim _{n, m} \frac{1}{n m} \sum_{j, k=0,0}^{\infty, \infty} a_{n m j k}\left[M_{j k}\left(\left\|\frac{u_{j k} \Delta_{s}^{r}\left(x_{j k}+y_{j k}\right)}{\rho}, z_{1}, \ldots, z_{n-1}\right\|\right)^{p_{j k}}\right] \\
&=\lim _{n, m} \frac{1}{n m} \sum_{j, k=0,0}^{\infty, \infty} a_{n m j k}\left[M_{j k}\left(\left\|\frac{u_{j k} \Delta_{s}^{r}\left(x_{j k}+y_{j k}\right)}{\rho_{1}+\rho_{2}}, z_{1}, \ldots, z_{n-1}\right\|\right)^{p_{j k}}\right] \\
&=\lim _{n, m} \frac{1}{n m} \sum_{j, k=0,0}^{\infty, \infty} a_{n m j k}\left[M_{j k}\left(\left\|\frac{u_{j k} \Delta_{s}^{r} x_{j k}}{\rho_{1}+\rho_{2}}, z_{1}, \ldots, z_{n-1}\right\|\right)^{p_{j k}}\right] \\
& \quad+\lim _{n, m} \frac{1}{n m} \sum_{j, k=0,0}^{\infty, \infty} a_{n m j k}\left[M_{j k}\left(\left\|\frac{u_{j k} \Delta_{s}^{r} y_{j k}}{\rho_{1}+\rho_{2}}, z_{1}, \ldots, z_{n-1}\right\|\right)^{p_{j k}}\right] \\
& \leq\left(\frac{\rho_{1}}{\rho_{1}+\rho_{2}}\right) \lim _{n, m} \frac{1}{n m} \sum_{j, k=0,0}^{\infty, \infty} a_{n m j k}\left[M_{j k}\left(\left\|\frac{u_{j k} \Delta_{s}^{r} x_{j k}}{\rho_{1}}, z_{1}, \ldots, z_{n-1}\right\|\right)^{p_{j k}}\right] \\
&+\left(\frac{\rho_{2}}{\rho_{1}+\rho_{2}}\right) \lim _{n, m} \frac{1}{n m} \sum_{j, k=0,0}^{\infty, \infty} a_{n m j k}\left[M_{j k}\left(\left\|\frac{u_{j k} \Delta_{s}^{r} y_{j k}}{\rho_{2}}, z_{1}, \ldots, z_{n-1}\right\|\right)^{p_{j k}}\right] \\
& \leq 1,
\end{aligned}
$$

and thus

$$
\begin{aligned}
& g(x+y) \\
& =\inf \left\{(\rho)^{\frac{p_{j k}}{M}}: \lim _{n, m} \frac{1}{n m} \sum_{j, k=0,0}^{\infty, \infty} a_{n m j k}\left[M_{j k}\left(\left\|\frac{u_{j k} \Delta_{s}^{r}\left(x_{j k}+y_{j k}\right)}{\rho}, z_{1}, \ldots, z_{n-1}\right\|\right)^{p_{j k}}\right]^{\frac{1}{M}} \leq 1\right\} \\
& \leq \inf \left\{\left(\rho_{1}\right)^{\frac{p_{j k}}{M}}: \lim _{n, m} \frac{1}{n m} \sum_{j, k=0,0}^{\infty, \infty} a_{n m j k}\left[M_{j k}\left(\left\|\frac{u_{j k} \Delta_{s}^{r} x_{j k}}{\rho_{1}}, z_{1}, \ldots, z_{n-1}\right\|\right)^{p_{j k}}\right]^{\frac{1}{M}} \leq 1\right\} \\
& \quad+\inf \left\{\left(\rho_{2}\right)^{\frac{p_{j k}}{M}}: \lim _{n, m} \frac{1}{n m} \sum_{j, k=0,0}^{\infty, \infty} a_{n m j k}\left[M_{j k}\left(\left\|\frac{u_{j k} \Delta_{s}^{r} y_{j k}}{\rho_{2}}, z_{1}, \ldots, z_{n-1}\right\|\right)^{p_{j k}}\right]^{\frac{1}{M}} \leq 1\right\} .
\end{aligned}
$$

Therefore, $g(x+y)=g(x)+g(y)$. 
Finally, we prove that the scalar multiplication is continuous. Let $\lambda$ be any complex number. By definition,

$$
\begin{aligned}
g(\lambda x) & =\inf \left\{(\rho)^{\frac{p_{j k}}{M}}: \lim _{n, m} \frac{1}{n m} \sum_{j, k=0,0}^{\infty, \infty} a_{n m j k}\left[M_{j k}\left(\left\|\frac{u_{j k} \Delta_{s}^{r} \lambda x_{j k}}{\rho}, z_{1}, \ldots, z_{n-1}\right\|\right)^{p_{j k}}\right]^{\frac{1}{M}} \leq 1\right\} \\
& =\inf \left\{(|\lambda| t)^{\frac{p_{j k}}{M}}: \lim _{n, m} \frac{1}{n m} \sum_{j, k=0,0}^{\infty, \infty} a_{n m j k}\left[M_{j k}\left(\left\|\frac{u_{j k} \Delta_{s}^{r} x_{j k}}{t}, z_{1}, \ldots, z_{n-1}\right\|\right)^{p_{j k}}\right]^{\frac{1}{M}} \leq 1\right\},
\end{aligned}
$$

where $t=\frac{\rho}{|\lambda|}>0$. Since $|\lambda|^{p_{j k}} \leq \max \left(1,|\lambda|^{\text {sup } p_{j k}}\right)$, we have

$$
\begin{aligned}
g(\lambda x) \leq & \max \left(1,|\lambda|^{\text {sup } p_{j k}}\right) \\
& \times \inf \left\{t^{p_{j k}}: \lim _{n, m} \frac{1}{n m} \sum_{j, k=0,0}^{\infty, \infty} a_{n m j k}\left[M_{j k}\left(\left\|\frac{u_{j k} \Delta_{s}^{r} x_{j k}}{t}, z_{1}, \ldots, z_{n-1}\right\|\right)^{p_{j k}}\right]^{\frac{1}{M}} \leq 1\right\} .
\end{aligned}
$$

So, the fact that the scalar multiplication is continuous follows from the above inequality. This completes the proof of the theorem.

Theorem 3.3 Let $\mathcal{M}=\left(M_{j k}\right)$ be a Musielak-Orlicz function, $A=\left(a_{n m j k}\right)$ be a nonnegative four-dimensional RH-regular matrix, $p=\left(p_{j k}\right)$ be a bounded sequence of positive real numbers and $u=\left(u_{j k}\right)$ be a sequence of strictly positive real numbers. Then $W_{0}^{2}\left(A, \mathcal{M}, u, \Delta_{s}^{r}, p,\|\cdot, \ldots, \cdot\|\right)$ and $W^{2}\left(A, \mathcal{M}, u, \Delta_{s}^{r}, p,\|\cdot, \ldots, \cdot\|\right)$ are complete topological linear spaces.

Proof Let $\left(x_{j k}^{q}\right)$ be a Cauchy sequence in $W_{0}^{2}\left(A, \mathcal{M}, u, \Delta_{s}^{r}, p,\|\cdot, \ldots, \cdot\|\right)$, that is, $g\left(x^{q}-x^{t}\right) \rightarrow 0$ as $q, t \rightarrow \infty$. Then we have

$$
\frac{1}{n m} \sum_{j, k=0,0}^{\infty, \infty} a_{n m j k}\left[M_{j k}\left(\left\|\frac{u_{j k} \Delta_{s}^{r} x_{j k}^{q}-u_{j k} \Delta_{s}^{r} x_{j k}^{t}}{\rho}, z_{1}, \ldots, z_{n-1}\right\|\right)^{p_{j k}}\right] \rightarrow 0 .
$$

Thus for each fixed $j$ and $k$ as $q, t \rightarrow \infty$, since $A=\left(a_{n m j k}\right)$ is nonnegative, we are granted that

$$
M_{j k}\left(\left\|\frac{u_{j k} \Delta_{s}^{r} x_{j k}^{q}-u_{j k} \Delta_{s}^{r} x_{j k}^{t}}{\rho}, z_{1}, \ldots, z_{n-1}\right\|\right) \rightarrow 0
$$

and by continuity of $\mathcal{M}=\left(M_{j k}\right),\left(x_{j k}^{q}\right)$ is a Cauchy sequence in $\mathbb{R}$ for each fixed $j$ and $k$.

Since $\mathbb{R}$ is complete as $t \rightarrow \infty$, we have $x_{j k}^{q} \rightarrow x_{j k}$ for each $(j, k)$. For $\epsilon>0$, there exists a natural number $N$ such that

$$
\lim _{n, m} \frac{1}{n m} \sum_{j, k=0,0 q, t>N}^{\infty, \infty} a_{n m j k}\left[M_{j k}\left(\left\|\frac{u_{j k} \Delta_{s}^{r} x_{j k}^{q}-u_{j k} \Delta_{s}^{r} x_{j k}^{t}}{\rho}, z_{1}, \ldots, z_{n-1}\right\|\right)^{p_{j k}}\right]<\epsilon \quad \text { for all } m, n \text {. }
$$

Since for any fixed natural number $M$, we have

$$
\lim _{n, m} \frac{1}{n m} \sum_{j, k \leq M q, t>N}^{\infty, \infty} a_{n m j k}\left[M_{j k}\left(\left\|\frac{u_{j k} \Delta_{s}^{r} x_{j k}^{q}-u_{j k} \Delta_{s}^{r} x_{j k}^{t}}{\rho}, z_{1}, \ldots, z_{n-1}\right\|\right)^{p_{j k}}\right]<\epsilon \quad \text { for all } m, n,
$$


and by letting $t \rightarrow \infty$ in the above expression we obtain

$$
\lim _{n, m} \frac{1}{n m} \sum_{j, k \leq M q>N}^{\infty, \infty} a_{n m j k}\left[M_{j k}\left(\left\|\frac{u_{j k} \Delta_{s}^{r} x_{j k}^{q}-u_{j k} \Delta_{s}^{r} x_{j k}}{\rho}, z_{1}, \ldots, z_{n-1}\right\|\right)^{p_{j k}}\right]<\epsilon .
$$

Since $M$ is arbitrary, by letting $M \rightarrow \infty$ we obtain

$$
\lim _{n, m} \frac{1}{n m} \sum_{j, k=0,0}^{\infty, \infty} a_{n m j k}\left[M_{j k}\left(\left\|\frac{u_{j k} \Delta_{s}^{r} x_{j k}^{q}-u_{j k} \Delta_{s}^{r} x_{j k}}{\rho}, z_{1}, \ldots, z_{n-1}\right\|\right)^{p_{j k}}\right]<\epsilon \quad \text { for all } m, n \text {. }
$$

Thus $g\left(x^{q}-x\right) \rightarrow 0$ as $q \rightarrow \infty$. This proves that $W_{0}^{2}\left(A, \mathcal{M}, u, \Delta_{s}^{r}, p,\|\cdot, \ldots, \cdot\|\right)$ is a complete topological linear space.

Now we shall show that $W^{2}\left(A, \mathcal{M}, u, \Delta_{s}^{r}, p,\|\cdot, \ldots, \cdot\|\right)$ is a complete topological linear space. For this, since $\left(x^{q}\right)$ is also a sequence in $W^{2}\left(A, \mathcal{M}, u, \Delta_{s}^{r}, p,\|\cdot, \ldots, \cdot\|\right)$ by definition of $W^{2}\left(A, \mathcal{M}, u, \Delta_{s}^{r}, p,\|\cdot, \ldots, \cdot\|\right)$, for each $q$ there exists $L^{q}$ with

$$
\frac{1}{n m} \sum_{j, k=0,0}^{\infty, \infty} a_{n m j k}\left[M_{j k}\left(\left\|\frac{u_{j k} \Delta_{s}^{r} x_{j k}^{q}-u_{j k} \Delta_{s}^{r} L^{q}}{\rho}, z_{1}, \ldots, z_{n-1}\right\|\right)^{p_{j k}}\right] \rightarrow 0 \quad \text { as } m, n \rightarrow \infty,
$$

whence, from the fact that $\sup _{n m} \frac{1}{n m} \sum_{j, k=0,0}^{\infty, \infty} a_{n m j k}<\infty$ and from the definition of a Musielak-Orlicz function, we have $M_{j k}\left(\left\|\frac{\Delta_{s}^{r} L^{q}-\Delta_{s}^{r} L}{\rho}, z_{1}, \ldots, z_{n-1}\right\|\right) \rightarrow 0$ as $q \rightarrow \infty$ and so $L^{q}$ converges to $L$. Thus

$$
\frac{1}{n m} \sum_{j, k=0,0}^{\infty, \infty} a_{n m j k}\left[M_{j k}\left(\left\|\frac{u_{j k} \Delta_{s}^{r} x_{j k}-L}{\rho}, z_{1}, \ldots, z_{n-1}\right\|\right)^{p_{j k}}\right] \rightarrow 0 \quad \text { as } m, n \rightarrow \infty .
$$

Hence $x \in W^{2}\left(A, \mathcal{M}, u, \Delta_{s}^{r}, p,\|\cdot, \ldots, \cdot\|\right)$ and this completes the proof.

Theorem 3.4 Let $\mathcal{M}=\left(M_{j k}\right)$ be a Musielak-Orlicz function which satisfies the $\Delta_{2}$ condition. Then $W^{2}\left(A, u, \Delta_{s}^{r}, p,\|\cdot, \ldots, \cdot\|\right) \subseteq W^{2}\left(A, \mathcal{M}, u, \Delta_{s}^{r}, p,\|\cdot, \ldots, \cdot\|\right)$.

Proof Let $x=\left(x_{k}\right) \in W^{2}\left(A, u, \Delta_{s}^{r}, p,\|\cdot, \ldots, \cdot\|\right)$, that is,

$$
\lim _{n, m} \frac{1}{n m} \sum_{j, k} a_{n m j k}\left[\left(\left\|\frac{u_{j k} \Delta_{s}^{r} x_{j k}-L}{\rho}, z_{1}, \ldots, z_{n-1}\right\|\right)^{p_{j k}}\right]=0 .
$$

Let $\epsilon>0$ and choose $\delta$ with $0<\delta<1$ such that $M_{j k}(t)<\epsilon$ for $0 \leq t \leq \delta$. Write $y_{j k}=$ $\left(\left\|\frac{u_{j k} \Delta_{s}^{r} x_{j k}-L}{\rho}, z_{1}, \ldots, z_{n-1}\right\|\right)$ and consider

$$
\begin{aligned}
& \lim _{n, m} \frac{1}{n m} \sum_{j, k} a_{n m j k}\left[M_{j k}\left(y_{j k}\right)^{p_{j k}}\right] \\
& =\lim _{n, m} \frac{1}{n m} \sum_{j, k:\left|y_{j k}\right| \leq \delta} a_{n m j k}\left[M_{j k}\left(y_{j k}\right)^{p_{j k}}\right]+\lim _{n, m} \frac{1}{n m} \sum_{j, k: 1 y_{j k} \mid>\delta} a_{n m j k}\left[M_{j k}\left(y_{j k}\right)^{p_{j k}}\right] \\
& =\epsilon \lim _{n, m} \frac{1}{n m} \sum_{j, k:\left|y_{j k}\right| \leq \delta} a_{n m j k}+\lim _{n, m} \frac{1}{n m} \sum_{j, k: 1 y_{j k} \mid>\delta} a_{n m j k}\left[M_{j k}\left(y_{j k}\right)^{p_{j k}}\right] .
\end{aligned}
$$


For $y_{j k}>\delta$, we use the fact that $y_{j k}<\frac{y_{j k}}{\delta}<1+\frac{y_{j k}}{\delta}$. Hence

$$
M_{j k}\left(y_{j k}\right)<M_{j k}\left(1+\frac{y_{j k}}{\delta}\right)<\frac{M_{j k}(2)}{2}+\frac{1}{2} M_{j k}\left(2 \frac{y_{j k}}{\delta}\right) .
$$

Since $\mathcal{M}$ satisfies the $\Delta_{2}$-condition, we have

$$
M_{j k}\left(y_{j k}\right)<K \frac{y_{j k}}{2 \delta} M_{j k}(2)+K \frac{y_{j k}}{2 \delta} M_{j k}(2)=K \frac{y_{j k}}{\delta} M_{j k}(2),
$$

and hence

$$
\begin{aligned}
& \lim _{n, m} \frac{1}{n m} \sum_{j, k:\left|y_{j k}\right|>\delta} a_{n m j k}\left[M_{j k}\left(y_{j k}\right)^{p_{j k}}\right] \\
& \quad \leq K \frac{M_{j k}}{\delta}(2) \lim _{n, m} \frac{1}{n m} \sum_{j, k} a_{n m j k}\left[\left(\left\|\frac{u_{j k} \Delta_{s}^{r} x_{j k}-L}{\rho}, z_{1}, \ldots, z_{n-1}\right\|\right)^{p_{j k}}\right] .
\end{aligned}
$$

Since $A$ is $R H$-regular and $x \in W^{2}\left(A, u, \Delta_{s}^{r}, p,\|\cdot, \ldots, \cdot\|\right)$, we get $x \in W^{2}\left(A, \mathcal{M}, u, \Delta_{s}^{r}, p\right.$, $\|\cdot, \ldots, \cdot\|)$.

Theorem 3.5 Let $\mathcal{M}=\left(M_{j k}\right)$ be a Musielak-Orlicz function and let $A=\left(a_{n m j k}\right)$ be a nonnegative four-dimensional RH-regular matrix. Suppose that $\beta=\lim _{t \rightarrow \infty} \frac{M_{j k}(t)}{t}<\infty$. Then

$$
W^{2}\left(A, u, \Delta_{s}^{r}, p,\|\cdot, \ldots, \cdot\|\right)=W^{2}\left(A, \mathcal{M}, u, \Delta_{s}^{r}, p,\|\cdot, \ldots, \cdot\|\right) .
$$

Proof In order to prove that $W^{2}\left(A, u, \Delta_{s}^{r}, p,\|\cdot, \ldots, \cdot\|\right)=W^{2}\left(A, \mathcal{M}, u, \Delta_{s}^{r}, p,\|\cdot, \ldots, \cdot\|\right)$. It is sufficient to show that $W^{2}\left(A, \mathcal{M}, u, \Delta_{s}^{r}, p,\|\cdot, \ldots, \cdot\|\right) \subset W^{2}\left(A, u, \Delta_{s}^{r}, p,\|\cdot, \ldots, \cdot\|\right)$. Now, let $\beta>0$. By definition of $\beta$, we have $M_{j k}(t) \geq \beta t$ for all $t \geq 0$. Since $\beta>0$, we have $t \leq \frac{1}{\beta} M_{j k}(t)$ for all $t \geq 0$. Let $x=\left(x_{j k}\right) \in W^{2}\left(A, \mathcal{M}, u, \Delta_{s}^{r}, p,\|\cdot, \ldots, \cdot\|\right)$. Thus, we have

$$
\begin{aligned}
& \lim _{n, m} \frac{1}{n m} \sum_{j, k=0,0}^{\infty, \infty} a_{n m j k}\left[\left(\left\|\frac{u_{j k} \Delta_{s}^{r} x_{j k}-L}{\rho}, z_{1}, \ldots, z_{n-1}\right\|\right)^{p_{j k}}\right] \\
& \leq \frac{1}{\beta} \lim _{n, m} \frac{1}{n m} \sum_{j, k=0,0}^{\infty, \infty} a_{n m j k}\left[M_{j k}\left(\left\|\frac{u_{j k} \Delta_{s}^{r} x_{j k}-L}{\rho}, z_{1}, \ldots, z_{n-1}\right\|\right)^{p_{j k}}\right],
\end{aligned}
$$

which implies that $x=\left(x_{j k}\right) \in W^{2}\left(A, u, \Delta_{s}^{r}, p,\|\cdot, \ldots, \cdot\|\right)$. This completes the proof.

\section{Theorem 3.6}

(i) Let $0<\inf p_{j k}<p_{j k} \leq 1$. Then

$$
W^{2}\left(A, \mathcal{M}, u, \Delta_{s}^{r}, p,\|\cdot, \ldots, \cdot\|\right) \subseteq W^{2}\left(A, \mathcal{M}, u, \Delta_{s}^{r},\|\cdot, \ldots, \cdot\|\right)
$$

(ii) Let $1 \leq p_{j k} \leq \sup p_{j k}<\infty$. Then

$$
W^{2}\left(A, \mathcal{M}, u, \Delta_{s}^{r},\|\cdot, \ldots, \cdot\|\right) \subseteq W^{2}\left(A, \mathcal{M}, u, \Delta_{s}^{r}, p,\|\cdot, \ldots, \cdot\|\right)
$$


Proof (i) Let $x=\left(x_{j k}\right) \in W^{2}\left(A, \mathcal{M}, u, \Delta_{s}^{r}, p,\|\cdot, \ldots, \cdot\|\right)$. Then since $0<\inf p_{j k}<p_{j k} \leq 1$, we obtain the following:

$$
\begin{aligned}
& \lim _{n, m} \frac{1}{n m} \sum_{j, k=0,0}^{\infty, \infty} a_{n m j k}\left[M_{j k}\left(\left\|\frac{u_{j k} \Delta_{s}^{r} x_{j k}-L}{\rho}, z_{1}, \ldots, z_{n-1}\right\|\right)\right] \\
& \quad \leq \lim _{n, m} \frac{1}{n m} \sum_{j, k=0,0}^{\infty, \infty} a_{n m j k}\left[M_{j k}\left(\left\|\frac{u_{j k} \Delta_{s}^{r} x_{j k}-L}{\rho}, z_{1}, \ldots, z_{n-1}\right\|\right)^{p_{j k}}\right] .
\end{aligned}
$$

Thus $x=\left(x_{j k}\right) \in W^{2}\left(A, \mathcal{M}, u, \Delta_{s}^{r},\|\cdot, \ldots, \cdot\|\right)$.

(ii) Let $p_{j k} \geq 1$ for each $j$ and $k$ and $\sup p_{j k}<\infty$. Let $x=\left(x_{j k}\right) \in W^{2}\left(A, \mathcal{M}, u, \Delta_{s}^{r},\|\cdot, \ldots, \cdot\|\right)$. Then for each $0<\epsilon<1$ there exists a positive integer $N$ such that

$$
\lim _{n, m} \frac{1}{n m} \sum_{j, k=0,0}^{\infty, \infty} a_{n m j k}\left[M_{j k}\left(\left\|\frac{u_{j k} \Delta_{s}^{r} x_{j k}-L}{\rho}, z_{1}, \ldots, z_{n-1}\right\|\right)\right] \leq \epsilon<1 \quad \text { for all } m, n \geq N .
$$

This implies that

$$
\begin{aligned}
& \lim _{n, m} \frac{1}{n m} \sum_{j, k=0,0}^{\infty, \infty} a_{n m j k}\left[M_{j k}\left(\left\|\frac{u_{j k} \Delta_{s}^{r} x_{j k}-L}{\rho}, z_{1}, \ldots, z_{n-1}\right\|\right)^{p_{j k}}\right] \\
& \leq \lim _{n, m} \frac{1}{n m} \sum_{j, k=0,0}^{\infty, \infty} a_{n m j k}\left[M_{j k}\left(\left\|\frac{u_{j k} \Delta_{s}^{r} x_{j k}-L}{\rho}, z_{1}, \ldots, z_{n-1}\right\|\right)\right] .
\end{aligned}
$$

Therefore $x=\left(x_{j k}\right) \in W^{2}\left(A, \mathcal{M}, u, \Delta_{s}^{r}, p,\|\cdot, \ldots, \cdot\|\right)$. This completes the proof.

Lemma 3.7 Let $G$ be an ideal in $l_{\infty}^{2}$ and let $x=\left(x_{j k}\right) \in l_{\infty}^{2}$. Then $x$ is in the closure of $G$ in $l_{\infty}^{2}$ if and only if $\chi_{S(x ; \epsilon)} \in G$ for all $\epsilon>0$.

Proof It is easy to prove so we omit the proof.

Lemma 3.8 Let $\mathcal{M}=\left(M_{j k}\right)$ be a Musielak-Orlicz function which satisfies the $\Delta_{2}$-condition and let $A=\left(a_{n m j k}\right)$ be a nonnegative four-dimensional $R H$-regular matrix. Then $W_{0}^{2}(A, \mathcal{M}$, $\left.u, \Delta_{s}^{r}, p,\|\cdot, \ldots, \cdot\|\right) \cap l_{\infty}^{2}$ is an ideal in $l_{\infty}^{2}$.

Proof Let $x \in W_{0}^{2}\left(A, \mathcal{M}, u, \Delta_{s}^{r}, p,\|\cdot, \ldots, \cdot\|\right) \cap l_{\infty}^{2}$ and $y \in l_{\infty}^{2}$. We need to show that $x y \in$ $W_{0}^{2}\left(A, \mathcal{M}, u, \Delta_{s}^{r}, p,\|\cdot, \ldots, \cdot\|\right) \cap l_{\infty}^{2}$. Since $y \in l_{\infty}^{2}$, there exists $T_{1}>1$ such that $\|y\|<T_{1}$. In this case $\left|x_{j k} y_{j k}\right|<T_{1}\left|x_{j k}\right|$ for all $j, k$. Since $\mathcal{M}$ is nondecreasing and satisfies $\Delta_{2}$-condition, we have

$$
\begin{aligned}
{\left[M_{j k}\left(\left\|\frac{u_{j k} \Delta_{s}^{r}\left(x_{j k} y_{j k}\right)}{\rho}, z_{1}, \ldots, z_{n-1}\right\|\right)^{p_{j k}}\right] } & <\left[M_{j k}\left(T_{1}\left\|\frac{u_{j k} \Delta_{s}^{r} x_{j k}}{\rho}, z_{1}, \ldots, z_{n-1}\right\|\right)^{p_{j k}}\right] \\
& \leq T\left(T_{1}\right)\left[M_{j k}\left(\left\|\frac{u_{j k} \Delta_{s}^{r} x_{j k}}{\rho}, z_{1}, \ldots, z_{n-1}\right\|\right)^{p_{j k}}\right]
\end{aligned}
$$

for all $j, k$, and $T>0$. Therefore $\lim _{n, m} \frac{1}{n m} \sum_{j, k} a_{n m j k}\left[M_{j k}\left(\left\|\frac{u_{j k} \Delta_{s}^{r}\left(x_{j k} y_{j k}\right)}{\rho}, z_{1}, \ldots, z_{n-1}\right\|\right)^{p_{j k}}\right]=0$. Thus, $x y \in W_{0}^{2}\left(A, \mathcal{M}, u, \Delta_{s}^{r}, p,\|\cdot, \ldots, \cdot\|\right) \cap l_{\infty}^{2}$. This completes the proof. 
Lemma 3.9 If $A$ is a nonnegative four-dimensional $R H$-regular matrix, then $W_{0}^{2}\left(A, u, \Delta_{s}^{r}\right.$, $p,\|\cdot, \ldots, \cdot\|) \cap l_{\infty}^{2}$ is a closed ideal in $l_{\infty}^{2}$.

Proof We have $W_{0}^{2}\left(A, \mathcal{M}, u, \Delta_{s}^{r}, p,\|\cdot, \ldots, \cdot\|\right) \cap l_{\infty}^{2} \subset l_{\infty}^{2}$ and it is clear that $W_{0}^{2}\left(A, \mathcal{M}, u, \Delta_{s}^{r}\right.$, $p,\|\cdot, \ldots, \cdot\|) \cap l_{\infty}^{2} \neq 0$. For $x, y \in W_{0}^{2}\left(A, \mathcal{M}, u, \Delta_{s}^{r}, p,\|\cdot, \ldots, \cdot\|\right) \cap l_{\infty}^{2}$, we get $\left|x_{j k}+y_{j k}\right|<$ $\left|x_{j k}\right|+\left|y_{j k}\right|$. Now, we have

$$
\begin{aligned}
& {\left[M_{j k}\left(\left\|\frac{u_{j k} \Delta_{s}^{r}\left(x_{j k}+y_{j k}\right)}{\rho}, z_{1}, \ldots, z_{n-1}\right\|\right)^{p_{j k}}\right]} \\
& \quad \leq\left[M_{j k}\left(\left\|\frac{u_{j k} \Delta_{s}^{r} x_{j k}}{\rho}, z_{1}, \ldots, z_{n-1}\right\|+\left\|\frac{u_{j k} \Delta_{s}^{r} y_{j k}}{\rho}, z_{1}, \ldots, z_{n-1}\right\|\right)^{p_{j k}}\right] \\
& \quad<\frac{1}{2}\left[M_{j k}\left(2\left\|\frac{u_{j k} \Delta_{s}^{r} x_{j k}}{\rho}, z_{1}, \ldots, z_{n-1}\right\|\right)^{p_{j k}}\right]+\frac{1}{2}\left[M_{j k}\left(2\left\|\frac{u_{j k} \Delta_{s}^{r} y_{j k}}{\rho}, z_{1}, \ldots, z_{n-1}\right\|\right)^{p_{j k}}\right] \\
& \quad<\frac{1}{2} K_{1}\left[M_{j k}\left(\left\|\frac{u_{j k} \Delta_{s}^{r} x_{j k}}{\rho}, z_{1}, \ldots, z_{n-1}\right\|\right)^{p_{j k}}\right]+\frac{1}{2} K_{2}\left[M_{j k}\left(\left\|\frac{u_{j k} \Delta_{s}^{r} y_{j k}}{\rho}, z_{1}, \ldots, z_{n-1}\right\|\right)^{p_{j k}}\right]
\end{aligned}
$$

by the $\Delta_{2}$-condition and the convexity of $M$. Since

$$
\begin{aligned}
\lim _{n, m} \frac{1}{n m} \sum_{j, k} a_{n m j k}\left[M_{j k}\left(\left\|\frac{u_{j k} \Delta_{s}^{r}\left(x_{j k}+y_{j k}\right)}{\rho}, z_{1}, \ldots, z_{n-1}\right\|\right)^{p_{j k}}\right] \\
\leq \frac{1}{2} K \lim _{n, m} \frac{1}{n m} \sum_{j, k} a_{n m j k}\left[M_{j k}\left(\left\|\frac{u_{j k} \Delta_{s}^{r} x_{j k}}{\rho}, z_{1}, \ldots, z_{n-1}\right\|\right)^{p_{j k}}\right] \\
\quad+\frac{1}{2} K \lim _{n, m} \frac{1}{n m} \sum_{j, k} a_{n m j k}\left[M_{j k}\left(\left\|\frac{u_{j k} \Delta_{s}^{r} y_{j k}}{\rho}, z_{1}, \ldots, z_{n-1}\right\|\right)^{p_{j k}}\right],
\end{aligned}
$$

where $K=\max \left\{K_{1}, K_{2}\right\}$, so $x+y, x-y \in W_{0}^{2}\left(A, \mathcal{M}, u, \Delta_{s}^{r}, p,\|\cdot, \ldots, \cdot\|\right) \cap l_{\infty}^{2}$.

Let $x \in W_{0}^{2}\left(A, \mathcal{M}, u, \Delta_{s}^{r}, p,\|\cdot, \ldots, \cdot\|\right) \cap l_{\infty}^{2}$ and $y \in l_{\infty}^{2}$. Thus, there exists a positive integer $K$, so that for every $j, k$, we have $\left|x_{j k} y_{j k}\right| \leq K\left|x_{j k}\right|$. Therefore

$$
\begin{aligned}
{\left[M_{j k}\left(\left\|\frac{u_{j k} \Delta_{s}^{r}\left(x_{j k} y_{j k}\right)}{\rho}, z_{1}, \ldots, z_{n-1}\right\|\right)^{p_{j k}}\right] } & \leq\left[M_{j k}\left(K\left\|\frac{u_{j k} \Delta_{s}^{r} x_{j k}}{\rho}, z_{1}, \ldots, z_{n-1}\right\|\right)^{p_{j k}}\right] \\
& \leq T\left[M_{j k}\left(\left\|\frac{u_{j k} \Delta_{s}^{r} x_{j k}}{\rho}, z_{1}, \ldots, z_{n-1}\right\|\right)^{p_{j k}}\right]
\end{aligned}
$$

and so

$$
\begin{aligned}
& \lim _{n, m} \frac{1}{n m} \sum_{j, k} a_{n m j k}\left[M_{j k}\left(\left\|\frac{u_{j k} \Delta_{s}^{r}\left(x_{j k} y_{j k}\right)}{\rho}, z_{1}, \ldots, z_{n-1}\right\|\right)^{p_{j k}}\right] \\
& \leq T \lim _{n, m} \frac{1}{n m} \sum_{j, k} a_{n m j k}\left[M_{j k}\left(\left\|\frac{u_{j k} \Delta_{s}^{r} x_{j k}}{\rho}, z_{1}, \ldots, z_{n-1}\right\|\right)^{p_{j k}}\right] .
\end{aligned}
$$

Hence $x y \in W_{0}^{2}\left(A, \mathcal{M}, u, \Delta_{s}^{r}, p,\|\cdot, \ldots, \cdot\|\right) \cap l_{\infty}^{2}$. So $W_{0}^{2}\left(A, \mathcal{M}, u, \Delta_{s}^{r}, p,\|\cdot, \ldots, \cdot\|\right) \cap l_{\infty}^{2}$ is an ideal in $l_{\infty}^{2}$ for a Musielak-Orlicz function which satisfies the $\Delta_{2}$-condition.

Now, we have to show that $W_{0}^{2}\left(A, \mathcal{M}, u, \Delta_{s}^{r}, p,\|\cdot, \ldots, \cdot\|\right) \cap l_{\infty}^{2}$ is closed. Let $x \in$ $\overline{W_{0}^{2}\left(A, \mathcal{M}, u, \Delta_{s}^{r}, p,\|\cdot, \ldots, \cdot\|\right) \cap l_{\infty}^{2}}$ there exists $x^{c d}=x_{j k}^{c d} \in W_{0}^{2}\left(A, \mathcal{M}, u, \Delta_{s}^{r}, p,\|\cdot, \ldots, \cdot\|\right) \cap l_{\infty}^{2}$ 
such that $x^{c d} \rightarrow x \in l_{\infty}^{2}$. For every $\epsilon>0$ there exists $N_{1}(\epsilon) \in \mathbb{N}$ such that for all $c, d>N_{1}(\epsilon)$, $\left|x^{c d}-x\right|<\epsilon$. Now, for $\epsilon>0$, we have

$$
\begin{aligned}
\lim _{n, m} & \frac{1}{n m} \sum_{j, k} a_{n m j k}\left[F_{j k}\left(\left\|\frac{u_{j k} \Delta_{s}^{r} x_{j k}}{\rho}, z_{1}, \ldots, z_{n-1}\right\|\right)^{p_{j k}}\right] \\
= & \lim _{n, m} \frac{1}{n m} \sum_{j, k} a_{n m j k}\left[M_{j k}\left(\left\|\frac{u_{j k} \Delta_{s}^{r} x_{j k}-u_{j k} \Delta_{s}^{r} x_{j k}^{c d}+u_{j k} \Delta_{s}^{r} x_{j k}^{c d}}{\rho}, z_{1}, \ldots, z_{n-1}\right\|\right)^{p_{j k}}\right] \\
\leq & \lim _{n, m} \frac{1}{n m} \sum_{j, k} a_{n m j k}\left[M _ { j k } \left(\left\|\frac{u_{j k} \Delta_{s}^{r} x_{j k}-u_{j k} \Delta_{s}^{r} x_{j k}^{c d}}{\rho}, z_{1}, \ldots, z_{n-1}\right\|\right.\right. \\
& \left.\left.+\left\|\frac{u_{j k} \Delta_{s}^{r} x_{j k}^{c d}}{\rho}, z_{1}, \ldots, z_{n-1}\right\|\right)^{p_{j k}}\right] \\
\leq & \frac{1}{2} \lim _{n, m} \frac{1}{n m} \sum_{j, k} a_{n m j k}\left[M_{j k}\left(2\left\|\frac{u_{j k} \Delta_{s}^{r} x_{j k}-u_{j k} \Delta_{s}^{r} x_{j k}^{c d}}{\rho}, z_{1}, \ldots, z_{n-1}\right\|\right)^{p_{j k}}\right] \\
& +\frac{1}{2} \lim _{n, m} \frac{1}{n m} \sum_{j, k} a_{n m j k}\left[M_{j k}\left(2\left\|\frac{u_{j k} \Delta_{s}^{r} x_{j k}^{c d}}{\rho}, z_{1}, \ldots, z_{n-1}\right\|\right)^{p_{j k}}\right] \\
\leq & \frac{1}{2} K M_{j k}(\epsilon) \lim _{n, m} \frac{1}{n m} \sum_{j, k} a_{n m j k} \\
& +\frac{1}{2} K \lim _{n, m} \frac{1}{n m} \sum_{j, k} a_{n m j k}\left[M_{j k}\left(\left\|\frac{u_{j k} \Delta_{s}^{r} x_{j k}^{c d}}{\rho}, z_{1}, \ldots, z_{n-1}\right\|\right)^{p_{j k}}\right] .
\end{aligned}
$$

Since $x^{c d} \in W_{0}^{2}\left(A, \mathcal{M}, u, \Delta_{s}^{r}, p,\|\cdot, \ldots, \cdot\|\right) \cap l_{\infty}^{2}$ and $A$ is RH-regular, we get

$$
\lim _{n, m} \frac{1}{n m} \sum_{j, k} a_{n m j k}\left[M_{j k}\left(\left\|\frac{u_{j k} \Delta_{s}^{r} x_{j k}}{\rho}, z_{1}, \ldots, z_{n-1}\right\|\right)^{p_{j k}}\right]=0
$$

so $x \in W_{0}^{2}\left(A, \mathcal{M}, u, \Delta_{s}^{r}, p,\|\cdot, \ldots, \cdot\|\right) \cap l_{\infty}^{2}$. This completes the proof.

Theorem 3.10 Let $x=\left(x_{j k}\right)$ be a bounded sequence, $\mathcal{M}=\left(M_{j k}\right)$ be a Musielak-Orlicz function which satisfies the $\Delta_{2}$-condition and $A$ be a nonnegative four-dimensional RH-regular matrix. Then $W^{2}\left(A, \mathcal{M}, u, \Delta_{s}^{r}, p,\|\cdot, \ldots, \cdot\|\right) \cap l_{\infty}^{2}=W^{2}\left(A, u, \Delta_{s}^{r}, p,\|\cdot, \ldots, \cdot\|\right) \cap l_{\infty}^{2}$.

Proof Without loss of generality we may take $L=0$ and establish

$$
W_{0}^{2}\left(A, \mathcal{M}, u, \Delta_{s}^{r}, p,\|\cdot, \ldots, \cdot\|\right) \cap l_{\infty}^{2}=W_{0}^{2}\left(A, u, \Delta_{s}^{r}, p,\|\cdot, \ldots, \cdot\|\right) \cap l_{\infty}^{2} .
$$

Since $W_{0}^{2}\left(A, u, \Delta_{s}^{r}, p,\|\cdot, \ldots, \cdot\|\right) \subseteq W_{0}^{2}\left(A, \mathcal{M}, u, \Delta_{s}^{r}, p,\|\cdot, \ldots, \cdot\|\right)$, therefore $W_{0}^{2}\left(A, u, \Delta_{s}^{r}, p\right.$, $\|\cdot, \ldots, \cdot\|) \cap l_{\infty}^{2} \subseteq W_{0}^{2}\left(A, \mathcal{M}, u, \Delta_{s}^{r}, p,\|\cdot, \ldots, \cdot\|\right) \cap l_{\infty}^{2}$. We need to show that $W_{0}^{2}\left(A, \mathcal{M}, u, \Delta_{s}^{r}\right.$, $p,\|\cdot, \ldots, \cdot\|) \cap l_{\infty}^{2} \subseteq W_{0}^{2}\left(A, u, \Delta_{s}^{r}, p,\|\cdot, \ldots, \cdot\|\right) \cap l_{\infty}^{2}$. Notice that if $S \subset \mathbb{N} \times \mathbb{N}$, then

$$
\lim _{n, m} \frac{1}{n m} \sum_{j, k} a_{n m j k}\left[M_{j k}\left(\chi_{S}(j, k)\right)^{p_{j k}}\right]=M_{j k}(1) \lim _{n, m} \frac{1}{n m} \sum_{j, k} a_{n m j k}\left(\chi_{S}(j, k)\right)^{p_{j k}},
$$


for all $n, m$. Observe that $\chi_{S}(j, k) \in W_{0}^{2}\left(A, u, \Delta_{s}^{r}, p,\|\cdot, \ldots, \cdot\|\right) \cap l_{\infty}^{2}$ whenever $x \in W_{0}^{2}(A, \mathcal{M}$, $\left.u, \Delta_{s}^{r}, p,\|\cdot, \ldots, \cdot\|\right) \cap l_{\infty}^{2}$ by Lemma 3.7 and Lemma 3.8, so

$$
W_{0}^{2}\left(A, \mathcal{M}, u, \Delta_{s}^{r}, p,\|\cdot, \ldots, \cdot\|\right) \cap l_{\infty}^{2} \subseteq W_{0}^{2}\left(A, u, \Delta_{s}^{r}, p,\|\cdot, \ldots, \cdot\|\right) \cap l_{\infty}^{2}
$$

The proof is complete.

\section{Competing interests}

The authors declare that they have no competing interests.

\section{Authors' contributions}

The authors contributed equally and significantly in writing this paper. The authors read and approved the final manuscript.

\section{Author details}

${ }^{1}$ Department of Mathematics, Faculty of Science, King Abdulaziz University, P.O. Box 80203, Jeddah, 21589, Saudi Arabia.

${ }^{2}$ School of Mathematics, Shri Mata Vaishno Devi University, Katra, Jammu and Kashmir 182320, India.

\section{Acknowledgements}

The authors gratefully acknowledge the financial support from King Abdulaziz University, Jeddah, Saudi Arabia.

Received: 20 April 2014 Accepted: 5 August 2014 Published: 2 September 2014

\section{References}

1. Gähler, S: Linear 2-normierte Räume. Math. Nachr. 28, 1-43 (1965)

2. Misiak, A: n-Inner product spaces. Math. Nachr. 140, 299-319 (1989)

3. Gunawan, $\mathrm{H}$ : On n-inner product, n-norms, and the Cauchy-Schwartz inequality. Sci. Math. Jpn. 5, 47-54 (2001)

4. Gunawan, H: The space of $p$-summable sequence and its natural n-norm. Bull. Aust. Math. Soc. 64, 137-147 (2001)

5. Gunawan, H, Mashadi, M: On n-normed spaces. Int. J. Math. Math. Sci. 27, 631-639 (2001)

6. Hardy, GH: On the convergence of certain multiple series. Proc. Camb. Philos. Soc. 19, 86-95 (1917)

7. Bromwich, TJ: An Introduction to the Theory of Infinite Series. Macmillan \& Co., New York (1965)

8. Móricz, F: Extension of the spaces $c$ and $c_{0}$ from single to double sequences. Acta Math. Hung. 57, 129-136 (1991)

9. Móricz, F, Rhoades, BE: Almost convergence of double sequences and strong regularity of summability matrices. Math. Proc. Camb. Philos. Soc. 104, 283-294 (1988)

10. Başarır, M, Sonalcan, O: On some double sequence spaces. J. Indian Acad. Math. 21, 193-200 (1999)

11. Mursaleen, M, Mohiuddine, SA: Regularly $\sigma$-conservative and $\sigma$-coercive four dimensional matrices. Comput. Math. Appl. 56, 1580-1586 (2008)

12. Mursaleen, M, Mohiuddine, SA: On $\sigma$-conservative and boundedly $\sigma$-conservative four-dimensional matrices. Comput. Math. Appl. 59, 880-885 (2010)

13. Mursaleen, M: Almost strongly regular matrices and a core theorem for double sequences. J. Math. Anal. Appl. 293(2), 523-531 (2004)

14. Mursaleen, $\mathrm{M}$, Edely, $\mathrm{OHH}$ : Almost convergence and a core theorem for double sequences. J. Math. Anal. Appl. 293(2), 532-540 (2004)

15. Altay, B, Başar, F: Some new spaces of double sequences. J. Math. Anal. Appl. 309, 70-90 (2005)

16. Başar, F, Sever, Y: The space $\mathcal{L}_{q}$ of double sequences. Math. J. Okayama Univ. 51, 149-157 (2009)

17. Mursaleen, M, Mohiuddine, SA: Some matrix transformations of convex and paranormed sequence spaces into the spaces of invariant means. J. Funct. Spaces Appl. 2012, Article ID 612671 (2012)

18. Mohiuddine, SA, Alotaibi, A: Some spaces of double sequences obtained through invariant mean and related concepts. Abstr. Appl. Anal. 2013, Article ID 507950 (2013)

19. Demirci, K: Strong A-summability and A-statistical convergence. Indian J. Pure Appl. Math. 27, $589-593$ (1996)

20. Mursaleen, M, Mohiuddine, SA: Some new double sequences spaces of invariant means. Glas. Mat. 45(65), 139-153 (2010)

21. Parashar, SD, Choudhary, B: Sequence spaces defined by Orlicz functions. Indian J. Pure Appl. Math. 25, 419-428 (1994)

22. Kizmaz, H: On certain sequences spaces. Can. Math. Bull. 24(2), 169-176 (1981)

23. Et, M, Çolak, R: On generalized difference sequence spaces. Soochow J. Math. 21(4), 377-386 (1995)

24. Et, M: Spaces of Cesàro difference sequences of order $r$ defined by a modulus function in a locally convex space. Taiwan. J. Math. 10(4), 865-879 (2006)

25. Et, M: Generalized Cesàro difference sequence spaces of non-absolute type involving lacunary sequences. Appl. Math. Comput. 219, 9372-9376 (2013)

26. Raj, K, Sharma, AK, Sharma, SK: A sequence space defined by Musielak-Orlicz function. Int. J. Pure Appl. Math. 67, 475-484 (2011)

27. Raj, K, Jamwal, S, Sharma, SK: New classes of generalized sequence spaces defined by an Orlicz function. J. Comput. Anal. Appl. 15, 730-737 (2013)

28. Raj, K, Sharma, SK: Some generalized difference double sequence spaces defined by a sequence of Orlicz-function. CUBO 14, 167-189 (2012) 
29. Raj, K, Sharma, SK, Sharma, AK: Some difference sequence spaces in n-normed spaces defined by Musielak-Orlicz function. Armen. J. Math. 3, 127-141 (2010)

30. Tripathy, BC: Generalized difference paranormed statistically convergent sequences defined by Orlicz function in a locally convex spaces. Soochow J. Math. 30, 431-446 (2004)

31. Et, M, Altin, Y, Choudhary, B, Tripathy, BC: On some classes of sequences defined by sequences of Orlicz functions. Math. Inequal. Appl. 9, 335-342 (2006)

32. Lindenstrauss, J, Tzafriri, L: On Orlicz sequence spaces. Isr. J. Math. 10, 379-390 (1971)

33. Maligranda, L: Orlicz Spaces and Interpolation. Seminars in Mathematics, vol. 5. Polish Academy of Science (1989)

34. Musielak, J: Orlicz Spaces and Modular Spaces. Lecture Notes in Mathematics, vol. 1034. Springer, Berlin (1983)

35. Pringsheim, A: Zur theorie der zweifach unendlichen zahlenfolgen. Math. Ann. 53, 289-321 (1900)

36. Cooke, RG: Infinite Matrices and Sequence Spaces. Macmillan \& Co., London (1950)

37. Robison, GM: Divergent double sequences and series. Trans. Am. Math. Soc. 28, 50-73 (1926)

38. Hamilton, HJ: Transformation of multiple sequences. Duke Math. J. 2, $29-60$ (1936)

39. Maddox, IJ: Elements of Functional Analysis, 2nd edn. Cambridge University Press, Cambridge (1988)

40. Simons, S: The sequence spaces $/\left(p_{v}\right)$ and $m\left(p_{v}\right)$. Proc. Lond. Math. Soc. 15(3), 422-436 (1965)

41. Yurdakadim, T, Tas, E: Double sequences and Orlicz functions. Period. Math. Hung. 67, 47-54 (2013)

42. Mohiuddine, SA, Raj, K, Alotaibi, A: Some paranormed double difference sequence spaces for Orlicz functions and bounded-regular matrices. Abstr. Appl. Anal. 2014, Article ID 419064 (2014)

doi:10.1186/1029-242X-2014-332

Cite this article as: Mohiuddine et al.: Generalized spaces of double sequences for Orlicz functions and bounded-regular matrices over n-normed spaces. Journal of Inequalities and Applications 2014 2014:332.

\section{Submit your manuscript to a SpringerOpen ${ }^{\circ}$ journal and benefit from:}

- Convenient online submission

Rigorous peer review

- Immediate publication on acceptance

- Open access: articles freely available online

- High visibility within the field

- Retaining the copyright to your article 